\title{
DELIRIO DE COTARD: PRESENTACIÓN DE CASO Y REVISIÓN DE LITERATURA
}

\section{Cotard Delusion: Case Presentation and Literature Review}

\author{
Manuel R. de Jesúsa , José A. Rojas ${ }^{b}$, Miguel Mejía Sangc, Vanesa Dislad, \\ Seily Nova ${ }^{e}$ Elvis González
}

Recibido: 6 de abril, 2020 • Aprobado: 6 de julio, 2020

Cómo citar: de Jesúsa MR, Rojas JA, Mejía Sang M, Disla V, Nova S, González E. Delirio de Cotard: presentación de caso y revisión de literatura. cysa [Internet]. 15 de febrero de 2021 [citado 23 de febrero de 2021];5(1):123-8. Disponible en: https://revistas.intec.edu. do/index.php/cisa/article/view/2054

\section{Resumen}

Introducción: el Síndrome o Delirio de Cotard (CS) es una condición psiquiátrica infrecuente caracterizada por la presencia de delirio de negación (o nihilista), donde el paciente refiere desde pérdida de capacidades intelectuales y expresión de sentimientos, hasta la negación de la propia existencia y la del cosmos. Fue descrito por primera vez por el neuropsiquiatra francés Jules Cotard en el ańo 1880.

El síntoma cardinal es el delirio nihilista, que consiste en un espectro de ideas de negación normalmente relacionadas con el cuerpo (negando la funcionabilidad o presencia de uno o más órganos internos) o con la existencia (pudiendo creer que está muerto o que todos en el mundo están muertos).

Presentación de caso: se realizó el reporte de un caso de un paciente masculino de 54 años de edad, proveniente de Los Hidalgos, Provincia Puerto Plata, República Dominicana, con antecedentes de diabetes mellitus, hipertensión y trastorno esquizoide no especificado. Dicho paciente

\footnotetext{
a Médico General. Santo Domingo, República Dominicana. ORCID: 0000-0001-6347-4148

Correo-e: m.dejesus1@prof.unibe.edu.do

b Médico General. Santo Domingo, República Dominicana.

Correo-e: joe.rojas1000@gmail.com

${ }^{c}$ Residente de Pediatría del Lincoln Medical and Mental Health Center, NY, EE. UU. Correo-e: mejiasm1@nychhc.org
}

\begin{abstract}
Introduction: Cotard Syndrome or Delirium (CS) is an infrequent psychiatric condition characterized by the presence of denial delirium (or nihilistic), where the patient refers from loss of intellectual abilities and expression of feelings, to denial of one's own existence and of the cosmos. It was first described by the French neuropsychiatrist Jules Cotard in the year 1880 .

The cardinal symptom is the nihilistic delirium, which consists of a spectrum of denial ideas normally related to the body (denying the functionality or presence of one or more internal organs) or to existence (being able to believe that they are dead or that everyone in the world is dead).

Case presentation: A case report was made of a 54-year-old male patient from Los Hidalgos, Puerto Plata Province, Dominican Republic, with a history of diabetes mellitus, hypertension and a nonspecified schizoid disorder. This patient presented the characteristic nihilistic delusions, as well as olfactory and auditory hallucinations, verbiage, psychomotor agitation, anorexia, insomnia and soliloquy.

\footnotetext{
d Psiquiatra del Zucker Hillside Hospital, Northwell Health. NY, EE. UU. Correo-e: vdisla1@northwell.edu

e Médico Internista del Hospital Central de las FFAA. Santo Domingo, República Dominicana. Correo-e: seyli_007@hotmail.com

${ }^{f}$ Psiquiatra del Hospital Central de las FFAA. Santo Domingo, Repú-
} blica Dominicana. Correo-e: gonzalezelvis@hotmail.com
\end{abstract}




\section{Manuel R. de Jesús, José A. Rojas, Miguel Mejía Sang, Vanesa Disla, Seily Nova y Elvis González}

presentó los característicos delirios nihilistas, además de alucinaciones olfativas y auditivas, verborrea, aumento de la motricidad, anorexia, insomnio y soliloquio.

A pesar de la limitada bibliografía, se pretendió comparar la presentación de dicho paciente con otros casos de Delirio de Cotard.

Palabras clave: delirio; delirio nihilista; trastorno esquizoide.

\section{Introducción}

El Síndrome o Delirio de Cotard (CS) es una condición psiquiátrica infrecuente caracterizada por la presencia de delirio de negación (o nihilista), donde el paciente refiere desde pérdida de capacidades intelectuales y expresión de sentimientos, hasta la negación de la propia existencia y la del cosmos. Fue descrito por primera vez por el neuropsiquiatra francés Jules Cotard en el año $1880^{1,2}$.

Este comprende cualquiera de una serie de delirios que van desde la creencia fija e inquebrantable de que se ha perdido órganos, sangre o partes del cuerpo hasta creer que se ha perdido el alma o está muerto. En su forma más profunda, el engaño toma la forma de una creencia profesa de que uno no existe. ${ }^{3}$ Se describen tres presentaciones: depresión psicótica (caracterizados por melancolía y pocos delirios nihilistas), Cotard tipo I (presenta tristeza patológica, ansiedad e ideas delirantes de tipos hipocondríaco y negación) y Cotard tipo II (configurado por alucinaciones auditivas, ansiedad, depresión y delirios de negación $)^{4}$.

El síntoma cardinal es el delirio nihilista, que consiste en un espectro de ideas de negación normalmente relacionadas con el cuerpo (negando la funcionabilidad o presencia de uno o más órganos internos) o con la existencia (pudiendo creer que está muerto o que todos en el mundo están muertos). Asimismo, esto puede conducir a delirios de inmortalidad, entendiendo que nada más puede matarlo, y deli-
Despite the limited bibliography, it was intended to compare the presentation of said patient with other cases of Cotard Delirium.

Keywords: delirium; nihilistic delirium; schizoid disorder.

rios hipocondríacos, donde el paciente entiende que sufre de una enfermedad severa o incurable ${ }^{5,6,7}$.

La presentación clínica del CS se acompaña además en un $89 \%$ de depresión, y en un $69 \%$ con ansiedad. Otros síntomas asociados incluyen: analgesia, automutilación, mutismo, inanición, ansiedad melancólica, ideas suicidas y alucinaciones. En estas últimas, las auditivas son las más frecuentes, pero también pueden ser olfativas o gustativas, donde perciben olor o sabor a materia fecal o putrefacción ${ }^{4,6,8-10}$.

Con respecto a la etiología y fisiopatología del CS, se debate si es un síndrome en sí mismo o síntoma de alguna otra condición. Actualmente no existe una definición del CS como tal en el DSM-V y el ICD $10^{5,6,11}$.

Debido la complejidad de los delirios de negación y distorsiones de autoimagen, se ha planteado la posibilidad de afección del lóbulo parietal, pero no se han reportado pruebas de laboratorio e imagen que corroboren esta teoría ${ }^{4,12}$.

Psicodinámicamente, las características del CS apuntan a una causa depresiva que produce sentimientos de culpa, desembocando en el autocastigo y en la total negación del mismo individuo, resultando en una distorsión entre el yo y el ello ${ }^{4}$.

Este síndrome puede ser primario, o puede ser secundario a numerosas condiciones, como depresión psicótica, esquizofrenia, desorden bipolar, 
demencia, neoplasia cerebral, hemorragia cerebral, y uso de sustancias psicoactivas ${ }^{4,12,13}$.

Además de esto, se han descrito asociaciones entre el CS y otros síndromes como el síndrome catatónico, síndrome neuroléptico maligno, licantropía, hidrofobia y síndrome de Capgras ${ }^{5,6}$.

El manejo del CS se basa en el tratamiento de la condición clínica orgánica subyacente, si existe. Se ha propuesto la utilización de la terapia electroconvulsiva como tratamiento de elección, debido a la presencia de delirios. Se utilizan antidepresivos si existen trastornos afectivos, y antipsicóticos si se acompaña de esquizofrenia ${ }^{4}$.

La vigilancia estricta del paciente es altamente recomendable debido al riesgo de autoagresión y suicidio, en especial en los pacientes con predominio de episodios depresivos ${ }^{4,8}$.

El pronóstico del CS es reservado. En los casos de intensidad moderada, la recuperación puede ser rápida o gradual. Sin embargo, aún en casos severos, se ha reportado recuperación súbita y espontánea. Normalmente, si el síndrome se encuentra acompañado de una condición orgánica subyacente, la recuperación depende de la evolución de dicha condición $^{4,12}$.

Cuando el CS se encuentra acompañado de síntomas depresivos, es posible la resolución de los mismos con permanencia del CS. Si se relaciona a síntomas psicóticos, ambos resuelven conjuntamente o pueden coexistir de forma crónica ${ }^{4}$.

Existen casos en que los pacientes perciben una doble orientación, en la que está consciente de su enfermedad y su pseudorrealidad se vuelve tolerable ${ }^{4}$.

\section{Reporte del caso}

Paciente masculino de 54 años de edad, proveniente de Los Hidalgos, Provincia Puerto Plata, República
Dominicana. Presenta antecedentes personales de hipertensión arterial sistémica y diabetes mellitus tipo 2 descompensado, de 1 mes y 6 meses de diagnosticados, respectivamente, y tratados de forma irregular con losartán/amlodipina y metformina/ glimepirida, de manera respectiva, y de un trastorno esquizoide inespecífico hace más de 20 años, caracterizado por aumento de la motricidad (caminaba largas distancias), ideas mesiánicas, irritabilidad, insomnio, hipofagia y verborrea, evaluado por psiquiatra y tratado con flufenazina de manera inconstante, con subsiguiente remisión y reinserción social y laboral. Conjuntamente, muestra historia de madre con trastorno psiquiátrico no especificado.

Hace 6 meses, paciente inició cuadro caracterizado por verborrea, aumento de la motricidad (caminaba repetidamente en círculos y alrededor de su casa, pretendiendo en ocasiones salir de la misma, pero quedándose parado frente a la puerta, o eventualmente salía y permanecía bajo el sol mirando fijamente el cielo), anorexia e incontinencia urinaria. La esposa del paciente describe que "habla solo" sin sonido audible "pero como si hablara con otra persona”. Paciente presenta delirios nihilistas (refiere que "está muerto" y que "le falta una pierna"), alucinaciones olfativas ("huele a muerto") y auditivas, sin ideaciones suicidas. Paciente fue evaluado por diferentes psiquiatras y tratado de forma irregular con quetiapina, ambulatoriamente.

Este cuadro clínico fue desencadenado por la muerte de un familiar a causa de envenenamiento. El paciente fue llevado por su esposa a nuestro centro por presentar agitación, verborrea e hipofagia (más de 10 días sin ingerir sólidos o líquidos, y más de 15 días sin tomar medicamentos).

Durante su estadía en el centro, el paciente se mantuvo orientado en persona y lugar, eulálico con volumen adecuado y latencia en respuesta, con facie tranquila, psicomotricidad conservada, insomnio y 


\section{Manuel R. de Jesús, José A. Rojas, Miguel Mejía Sang, Vanesa Disla, Seily Nova y Elvis González}

alucinaciones auditivas y visuales. En su segundo día de ingreso, paciente presentó crisis de ausencia.

El paciente se mantuvo bajo medicación con risperidona, carbamazepina, haloperidol, litio, diazepam, difenhidramina, insulina cristalina y metformina.

Luego de ser evaluado por los departamentos de Psiquiatría, Medicina Interna, y Endocrinología, presenta respuesta al manejo terapéutico, mejoría de su cuadro clínico sin eventualidades. Tomografía Axial Computarizada (TAC) de cráneo negativa, se decide su egreso a los 13 días de ingreso.

\section{Discusión}

Los trastornos psiquiátricos son comunes; a nivel mundial, una de cada tres personas se ha visto afectada en algún punto de su vida. El síndrome de Cotard es una entidad clínica infrecuente cuya principal característica es el delirio de negación. Actualmente no existe un consenso en la definición del síndrome, por lo que no cuenta con una descripción específica en el Manual Diagnóstico y Estadístico de los Trastornos Mentales (DSM-5) ${ }^{1,5,6,8,14}$.

Por esta razón, se ha imposibilitado realizar estudios formales que arrojen información veraz y concreta del Delirio de Cotard, por lo que existe poca documentación acerca del mismo.

Con respecto del caso reportado, el paciente mostraba los característicos delirios nihilistas, además de alucinaciones olfativas y auditivas, verborrea, aumento de la motricidad, anorexia, insomnio y soliloquio. Estos rasgos se asemejan al caso descrito por Machado et al. (2016) de un paciente de 31 años de edad que mostraba insomnio, anorexia, soliloquio, intento de suicidio, hipoprosexia, estado depresivo, retraso psicomotor y bradipsiquia. El paciente presentaba delirios de negación (reportaba estar vacío por dentro, pues sus órganos habían sido destruidos, y que estaba podrido), hipocondríaco (decía que su cuerpo estaba deformado y su cara llena de hoyos) y de culpa. Fue tratado con imipramina y risperidona, alcanzando recuperación clínica y reinserción social luego de 60 días en la institución 5 .

Con relación a los antecedentes psiquiátricos, el caso reportado no cuenta con historia de estado depresivo, incluyéndose en el $11 \%$ de casos del síndrome de Cotard que no presentan antecedentes de este tipo, de acuerdo con Castrillón Muñoz et al. (2009). Estos exponen un caso acerca de una paciente de 48 años que presenta antecedentes depresivos conjuntamente con cuadro clínico caracterizado por insomnio, hiporexia, anhedonia, tristeza, además de ideas de culpa, desesperanza y minusvalía, y contemplación de suicidio. Asimismo, presenta alucinaciones visuales (veía humo que salía por su boca, que interpretaba que era su alma saliendo de su cuerpo, por lo que decía estar "muerta en vida"), olfatorias (olor a podrido) y cenestésicas (parestesias que interpretaba como señales de que su carne estaba en descomposición). Fue tratada inicialmente con haloperidol, luego olanzapina y terapia convulsiva, lográndose mejoría de sus síntomas con ácido valproico, luego de un mes de internamiento ${ }^{4}$.

De la misma manera, Carano et al. (2014) presentaron un caso de una paciente femenina de 45 años de edad con historia de síntomas disociativos, afecto plano, estado depresivo, ansiedad, insomnio, irritabilidad, esquizofrenia paranoide. Además, presentaba delirios de negación (contaba que había perdido todo, no tenía médula espinal, ni cerebro, no podía llorar porque no tenía lágrimas, no tenía pulmones, intestinos, piernas, ni dientes, que su cuerpo estaba vacío y petrificado) y culpabilidad. Mostraba una TAC cerebral exhibiendo aumento ventricular y del líquido cefalorraquídeo, sin lesiones focales en parénquima. Fue tratado con diazepam, aripiprazol y sertralina, con recuperación completa en dos semanas ${ }^{6}$.

Tomando en consideración la imprecisión de los estudios de imágenes y laboratorio como métodos diagnósticos para el CS, Charland-Verville et al. (2013) 
expusieron el caso un masculino de 48 años de edad con diagnóstico de Síndrome de Cotard y estado depresivo con ideaciones suicidas, que fue sujeto a una tomografía con emisión de positrones (TEP) para evaluar el metabolismo cerebral. Evidenciaron un hipometabolismo bilateral en la corteza frontoparietal, e hipermetabolismo en el cerebelo, tallo cerebral y tálamo. Concluyeron a partir de esto, que el CS afecta las áreas del cerebro asociadas a la alerta consciente y en la integración del yo ${ }^{15}$.

$\mathrm{Al}$ paciente del caso reportado le fue realizado una TAC de cráneo que no evidenció cambios patológicos, por lo que inferimos que una TEP pudiera arrojar luz en el diagnóstico de esta entidad clínica, para ser identificada de manera temprana y manejada oportunamente.

\section{Conclusión}

El Síndrome de Cotard, como muchas condiciones psiquiátricas, puede tener causas somáticas y psiquiátricas, por lo que es un diagnóstico de exclusión que requiere una investigación exhaustiva del paciente, una descripción detallada de sus antecedentes y episodios anteriores, y un tratamiento oportuno para lograr disminuir la mortalidad debido a la alta tasa de suicidios asociados con la misma.

\section{Bibliografía}

1. Oberndorfer R, Schönauer C, Eichbauer H, Klaushofer K, Friedrich, F. Cotard Syndrome in Hypoactive Delirium - a Case Report. Psychiatr Danub. 2017;29(4):500-2.

2. Helldén A, Odar-Cederlöf I, Larsson K, Fehrman-Ekholm I, Lindén T. Death Delusion. BMJ. 2007;335:1305.

3. Pearn J, Gardner-Thorpe C.Jules Cotard (18401889): his life and the unique syndrome which bears his name. Neurology. 2003;58(9):14001403.
4. Castrillón Muñoz E, Gutiérrez Alzate B. Síndrome de Cotard: presentación de un caso. Rev Colomb Psiquiat. 2009;38(1):194-202.

5. Machado L., Lima Filho LE., Machado L. When the Patient Believes That the Organs Are Destroyed: Manifestation of Cotard's Syndrome [Internet]. Case Reports in Medicine. 2016;1-3. Disponible en: http://dx.doi. org/10.1155/2016/5101357

6. Carano A, De Berardis D, Cavuto M, Ortolani C, Perna G, Valchera A, et al. Cotard's Syndrome: Clinical Case Presentation and Literature Review. Int Neuropsychiatr Dis J. 2014;2(6):348-55.

7. Moschopoulos NP, Kaprinis S, Nimatoudis J. Psychiatriki. 2016;27(4):296-302. Doi:10. 22365/jpsych.2016.274.296

8. Silva JA, Leong GB, Weinstock R, Gonzales CL. A case of Cotard's syndrome associated with self-starvation. J Forens Sci. 2000;45:188-90

9. Grover S, Aneja J, Mahajan S, Varma S. Cotard's syndrome: Two case reports and a brief review of literature. J Neurosci Rural Pract. 2014;5(Suppl 1):S59-S62.

10. Huarcaya-Victoria J, Ledesma-Gastañadui M, Huete-Cordova M. Cotard's Syndrome in a Patient with Schizophrenia: Case Report and Review of the Literature. Case Rep Psychiatry. 2016;2016:ID6968409. Available from https:// doi.org/10.1155/2016/6968409

11. American Psychiatric Association. Diagnostic and Statistical Manual of Mental Disorders. American Psychiatric Association, Washingotn DC, USA. 2013; 5th edition.

12. Fuke T, Takahashi T, Yamada Y, Miyashita M, Amano N, Matsushita M. Seishin Shinkeigaku Zasshi. 2015;117(4):257-68. 


\section{Manuel R. de Jesús, José A. Rojas, Miguel Mejía Sang, Vanesa Disla, Seily Nova y Elvis González}

13. Debruyne H, Audenaert K. Towards understanding Cotard's syndrome: an overview. Neuropsychiatry. 2012;2(6):481-6.

14. Organización Mundial de la Salud. Cross-national comparisons of the prevalences and correlates of mental disorders. WHO International Consortium in Psychiatric Epidemiology. Bull World Health Org. 2000;78(4):413-26.
15. Charland-Verville V, Bruno MA, Ali Bahri M, Demertzi A, Desseilles $M$, Chatelle $C$, et al. Brain dead yet mind alive: A positron emission tomography case study of brain metabolism in Cotard's syndrome. Cortex. 2013;49(7):1997-9. 